\title{
Impact of LoRa Imperfect Orthogonality: Analysis of Link-level Performance
}

\author{
Daniele Croce, Michele Gucciardo, Stefano Mangione, Giuseppe Santaromita, Ilenia Tinnirello
}

\begin{abstract}
In this letter we focus on the evaluation of linklevel performance of LoRa technology, in the usual network scenario with a central gateway and high-density deployment of end-devices. LoRa technology achieves wide coverage areas, low power consumption and robustness to interference thanks to a chirp spread-spectrum modulation, in which chirps modulated with different spreading factors (SFs) are quasi-orthogonal. We focus on the performance analysis of a single receiver in presence of collisions. First, we analyze LoRa modulation numerically and show that collisions between packets modulated with different SFs can indeed cause packet loss if the interference power received is strong enough. Second, we validate such findings in experiments based on commercial devices and softwaredefined radios. Contradicting the common belief that SFs can be considered orthogonal, our results demonstrate that interSF collisions are indeed an issue in LoRa networks and, thus, allocating higher SFs to users far from the gateway might not necessarily improve their link capacity, in case of congested networks.
\end{abstract}

Index Terms-LoRa, spreading factor, interference.

\section{INTRODUCTION}

Recent years have seen an impressive proliferation of wireless technologies and mobile-generated traffic, which is now the highest portion of the total Internet traffic and is expected to grow further with the emergence of Internet-of-Things (IoT) applications [1]. Such a proliferation has been characterized by an high-density deployment of base stations (based on heterogeneous technologies, such as $4 \mathrm{G}$ cellular base stations and WiFi Access Points), as well as by high-density wireless devices, not limited to traditional user terminals. Indeed, with the advent of IoT applications, many smart objects, such as domestic appliances, cameras, monitoring sensors, etc., are equipped with a wireless technology.

In this paper we consider the emerging LoRa technology, which represents a critical example of wireless technology working with high-density networks. Indeed, LoRa has been conceived for Low Power Wide Area Networks (LPWAN), characterized by low data rate requirements per single device, large cells and heterogeneous application domains, which may lead to extremely high numbers of devices coexisting in the same cell. For this reason, LoRa provides different possibilities to orthogonalize transmissions as much as possible - Carrier Frequency (CF), Spreading Factor (SF), Bandwidth (BW), Coding Rate $(\mathrm{CR})$ - and provide simultaneous collision free

Manuscript received XXXX; accepted XXXX. Date of publication XXXX; date of current version XXXX. This work has been partially supported by EU funded research project symbIoTe, H2020-ICT-2015 grant agreement 688156. The associate editor coordinating the review of this letter and approving it for publication was XXXX. The authors are with the Department of Electrical Engineering, Università di Palermo, 90133 Palermo, Italy, and the CNIT Consortium, Italy (Corresponding author: Daniele Croce; e-mail: daniele.croce@cnit.it). Digital Object Identifier XXXXXXX. communications. However, despite of the robustness of the LoRa PHY [2] patented by Semtech [3], in WAN scenarios where multiple gateways can be installed, the scalability of this technology is still under investigation [4]. Current studies are mostly based on simulation results [5] and assume that the utilization of multiple transmission channels and SFs lead to a system that can be considered as the simple super-position of independent (single channel, single SF) sub-systems. This is actually a strong simplification, especially because the SFs adopted by LoRa are quasi-orthogonal [6] and therefore, in near-far conditions, collisions can prevent the correct reception of the overlapping transmissions using different SFs. The paper in [7] quantifies the power reception thresholds for different modulation formats and the Signal-to-Interference-Ratio (SIR) required for rejecting interfering LoRa signals, modulated with different spreading factors. However, no justification about the derivation of these numbers is provided and, as we will show, their theoretical results are very different from our experimental ones.

In this letter, we analyze LoRa modulation both numerically and experimentally, showing that collisions between packets of different SFs can indeed cause packet loss. We model the receiver performance of a reference device receiving a useful LoRa signal, under the presence of potential interfering signals generated by other end-devices or gateways. We quantify the Signal-to-Interference Ratio (SIR) values for which interference rejection of other LoRa signals does not work, for all combinations of SFs. To this purpose, we developed both a LoRa PHY simulator in MATLAB (for theoretical analysis) and a software transceiver able to generate LoRa modulated packets and to process a real LoRa signal (synthesized in software or acquired by means of the well known USRP software-defined-radio SDR platform). The source code of the simulator and some sample USRP traces can be found at [8]. The transceiver has been integrated in a traffic generator for LoRa networks, able to create, in a controlled and repeatable manner, a combined radio signal given by the super-position of multiple LoRa signals produced by different devices - and different SFs. We use the traffic generator for experimentally characterize the data extraction rate of a real receiver, when multiple links are simultaneously active. Our experimental results show that the SIR threshold for receiving a packet correctly is almost independent of the SF, with an average cochannel rejection of $-16 \mathrm{~dB}$. This has important implications for LoRa operators and network planning professionals: allocating higher SFs to far users could not necessarily improve their link capacity in case of congested networks because these transmissions are then received at lower power and are very prone to collisions due to longer transmission times. 

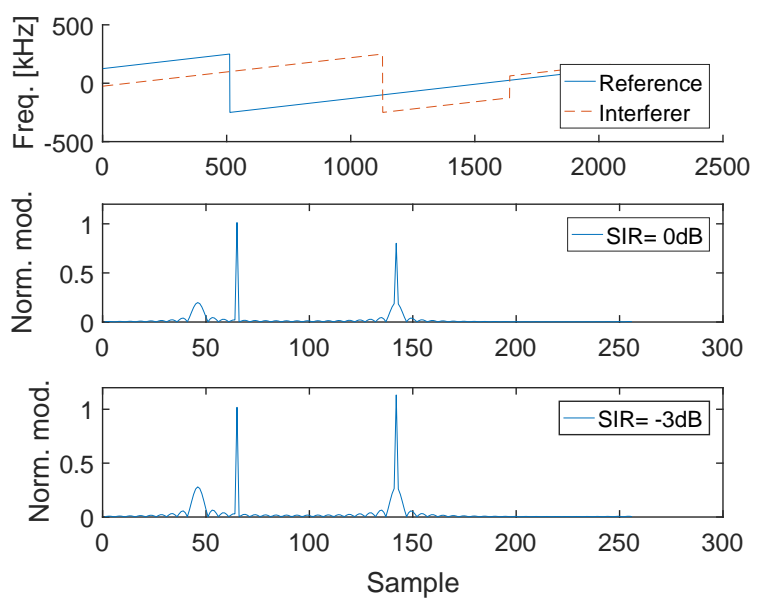

Figure 1. Capture effect of signals modulated with same SF. A LoRa reference symbol (top diagram, solid line) and two interfering symbols with the same SF (dashed line) are received at different SIRs, leading to correct reception (center diagram) or wrong detection of the symbol (bottom diagram).

\section{DisSECTING LoRA}

\section{A. LoRa modulation and demodulation}

LoRa modulation is derived from Chirp Spread Spectrum (CSS), which makes use of chirp signals, i.e. frequencymodulated signals obtained when the modulating signal varies linearly in the range $\left[f_{0}, f_{1}\right]$ (up-chirp) or $\left[f_{1}, f_{0}\right]$ (downchirp) in a symbol time $T$. LoRa employs a M-ary modulation scheme based on chirps, in which symbols are obtained by considering different circular shifts of the basic up-chirp signal. The temporal shifts, characterizing each symbol, are slotted into multiples of time $T_{\text {chip }}=1 / B W$, called chip, being $B W=f_{1}-f_{0}$ the bandwidth of the signal. It results that the modulating signal for a generic $n$-th LoRa symbol can be expressed as:

$$
f(t)= \begin{cases}f_{1}+k\left(t-n \cdot T_{\text {chip }}\right) & \text { for } 0 \leqslant t \leqslant n \cdot T_{\text {chip }} \\ f_{0}+k\left(t-n \cdot T_{\text {chip }}\right) & \text { for } n \cdot T_{\text {chip }}<t \leqslant T\end{cases}
$$

where $k=\left(f_{1}-f_{0}\right) / T$ is the slope of the frequency variations. The total number of symbols (coding $i$ information bits) is chosen equal to $2^{i}$, where $i$ is the SF. The symbol duration $T$ required for representing any possible shift is $2^{i} \cdot T_{\text {chip }}=$ $2^{i} / B W$. It follows that, for a fixed bandwidth, the symbol period and the temporal occupancy of the signal increase with larger SFs. The preamble of any LoRa frame is obtained by sending a sequence of at least eight up-chirps followed by two coded up-chirps, used for network identification (sync word), and two and a quarter base down-chirps. Payload data are then sent by using the M-ary modulation symbols. LoRa provides three $B W$ settings $(125,250$ or $500 \mathrm{kHz})$ and seven different $\mathrm{SF}$ values (from 6 to 12). In general, a larger bandwidth translates in a data rate increase and a receiver sensitivity deterioration. Conversely, higher SFs can be used to improve the link robustness at the cost of lower data rates.

An interesting feature of LoRa modulation is the quasiorthogonality of signals modulated under different SFs. This feature can be exploited for enabling multiple concurrent transmissions, thanks to the fact that the cross-energy between two non-synchronized signals modulated with different SFs
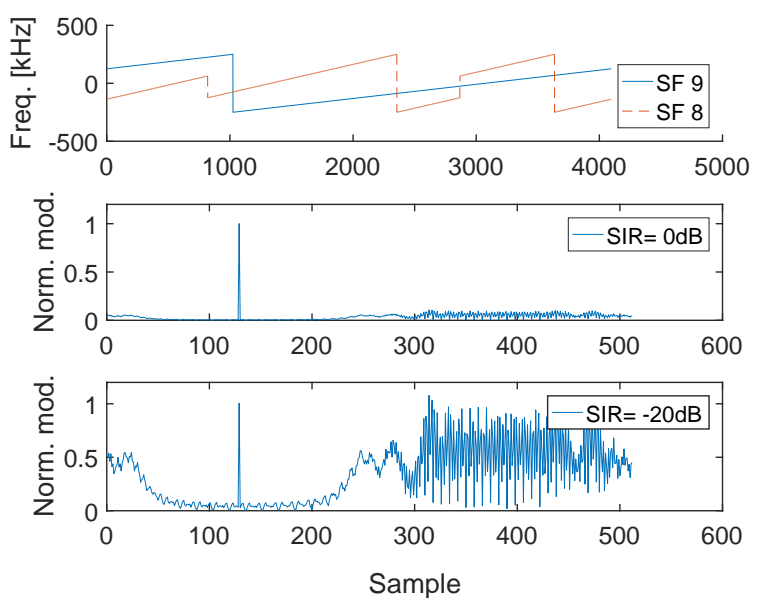

Figure 2. Collision example of signals modulated with different SFs. A LoRa symbol with SF 9 (top diagram, solid line) and two interfering symbols with SF 8 (dashed line) are received at different SIRs, leading to correct reception (center diagram) or wrong detection of the symbol (bottom diagram).

is almost zero. LoRa demodulation at the end-devices can be implemented with a very simple receiver architecture [3]. The receiver multiplies the received signal to the synchronized base down-chirp for obtaining a signal comprising only two frequencies: $f_{n}=-k n \cdot T_{\text {chip }}$ and $f_{n}-B W=-\left(f_{1}-\right.$ $\left.f_{0}\right)-k n \cdot T_{\text {chip }}$. Both frequencies can be aliased to the same frequency $f_{n}$ by down-sampling at the rate $B W$. Finally, the symbol index $\hat{n}$ can be estimated by considering the position of the peak at the output of an iFFT, as described in [7].

In case the received signal is given by the collision of two LoRa modulated signals (as shown in Fig. 1 and Fig. 2), we can distinguish two different scenarios, depending on the interfering spreading factor $S F_{\text {int }}$. First, if the $S F_{\text {int }}$ is the same as the one the receiver is listening for, the above receiver will observe multiple peaks at the output of the iFFT. As shown in Fig 1 assuming that the two transmissions are received at the same power and that the reference signal is perfectly synchronized with the receiver, the iFFT will show a maximum peak corresponding to the reference symbol and two smaller peaks corresponding to two partially overlapping interference symbols. A SIR of $0 \mathrm{~dB}$ can be sufficient for avoiding ambiguities in the identification of the maximum peak of the reference signal and for allowing to "capture" the channel. This means that LoRa exhibits a very high capture probability with the same SF. Second, when the $S F_{\text {int }}$ is different from the one the receiver is interested in, after multiplication with the base down-chirp and downsampling, the interfering signal will still be a chirped waveform, resulting in a wide-band spectrum with low spectral density, as shown in Fig. 2. Since the receiver estimates the transmitted symbol by looking for a peak, the co-channel rejection in this scenario results much higher, i.e. errors can occur at very low SIR values $(\approx-20 \mathrm{~dB}$ in the figure).

\section{B. LoRa PHY coding}

Up to now, we have neglected the impact of bit coding schemes. Indeed, the patented LoRa PHY includes several mechanisms to make the system more robust to interference. After the preamble, both header and payload bits of LoRa 

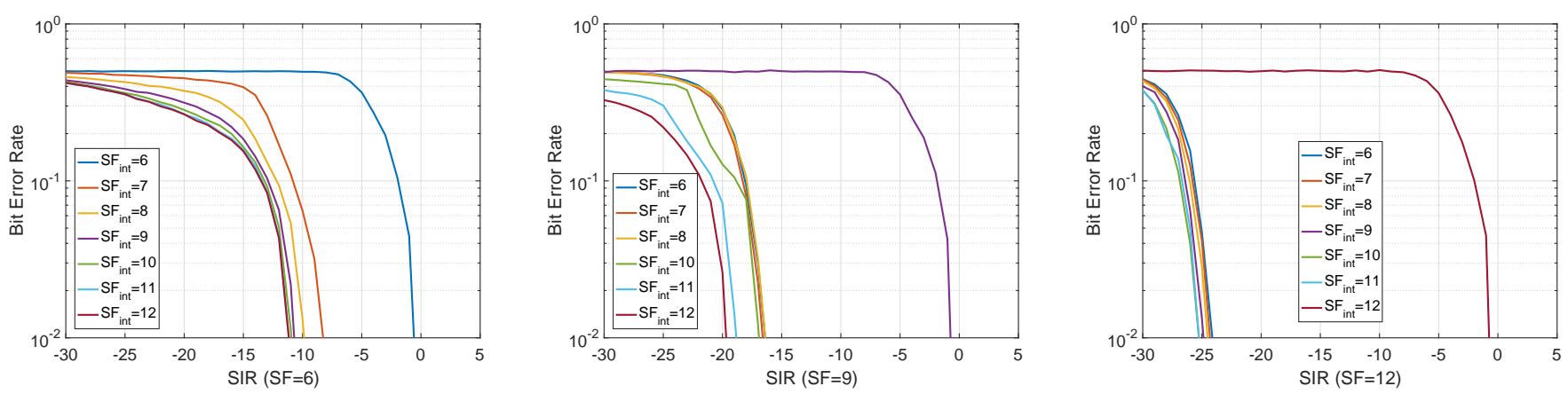

Figure 3. BER of three different spreading factors in function of the SIR.

frames are mapped to symbols by a pipeline of processing operations, which include: parity check or Hamming coding (rate $4 / 5$ to $4 / 8$ ), whitening, shuffling \& interleaving, and Gray coding. These operations have been specifically designed for increasing robustness towards synchronization errors or narrowband interference, which can be a serious issue for CSS-based modulations. In fact, in case of synchronization errors or narrowband interference, the receiver described in the previous section will most probably mistake the transmitted symbol, mapped to frequency $f_{n}$ after the iFFT, for one of the immediately adjacent symbols. Since gray coding ensures that adjacent symbols are mapped to bit patterns differing in one position only, the receiver is able to identify the less reliable bits (at most two bits) of each received symbol. The purpose of the LoRa interleaver is spreading unreliable bits among several codewords, thus enabling even the 4/5 Hamming code (consisting in a simple parity check) in exhibiting a significant channel coding gain.

In order to understand if Gray coding has an impact also on inter-SF interference, we tried to characterize the distance between the transmitted symbol and the decoded one in presence of inter-SF collisions. To this purpose, we extended our MATLAB implementation with Gray encoding and quantified such distance in our simulation. From our experiments we have seen that the error distance probability approximates a Binomial distribution and is not concentrated around the adjacent symbol. Thus, LoRa PHY coding mechanisms can mitigate synchronization errors but cannot protect from collisions.

\section{RESULTS}

\section{A. MATLAB simulations}

To quantify the co-channel rejection, including the impact of PHY coding, we implemented a LoRa modulator and demodulator in MATLAB, based on [3] and [7]. We performed a number of simulations for testing the reception of two overlapping transmissions modulated with different SFs, after Hamming coding at rate 4/7, interleaving and Gray encoding. Our goal is identifying a SIR threshold below which the demodulation of the received frame is affected by errors. In each simulation run, we created an overlapped signal by summing the reference frame, modulated with a reference spreading factor $S F_{\text {ref }}$, with a number of random interfering symbols, modulated with a different spreading factor $S F_{\text {int }}$ (with an equivalent time on air). We assumed the transmitter to be perfectly synchronized with the receiver, while
Table I

SIR THRESHOLDS IN MATLAB SIMULATIONS.

\begin{tabular}{|r|r|r|r|r|r|r|r|}
\hline$S F_{\text {int }}$ & 6 & 7 & 8 & 9 & 10 & 11 & 12 \\
\hline 6 & 0 & -8 & -10 & -11 & -11 & -11 & -11 \\
\hline 7 & -11 & 0 & -11 & -13 & -14 & -14 & -14 \\
\hline 8 & -14 & -13 & 0 & -14 & -16 & -17 & -17 \\
\hline 9 & -17 & -17 & -16 & 0 & -17 & -19 & -20 \\
\hline 10 & -19 & -19 & -19 & -19 & 0 & -20 & -22 \\
\hline 11 & -22 & -22 & -22 & -22 & -22 & 0 & -23 \\
\hline 12 & -24 & -24 & -24 & -25 & -25 & -25 & 0 \\
\hline
\end{tabular}

the interference frame is randomly shifted in time for desynchronizing the interfering symbols. The amplitude $A_{\text {ref }}$ of the reference signal is set to one, whereas the amplitude $A_{\text {int }}$ of the interferer is a tunable value depending on the SIR, i.e. $A_{\text {int }}=\sqrt{10^{-S I R / 10}} \cdot A_{\text {ref }}$. The resulting combined signal has been then processed by the MATLAB demodulator, in absence of noise on the channel. For each simulation run, we randomly generated interfered packets until the occurrence of 100 total error events. Packets are transmitted with $S F_{\text {ref }}$ and include 20 Bytes of data and a zero padding up to an integer number of interleaving blocks. This signal is interfered by a random LoRa-like signal modulated with $S F_{\text {int }}$, with a random time-offset and a SIR increasing from $-30 \mathrm{~dB}$ with $1 \mathrm{~dB}$ steps. A Bit Error Rate (BER) statistic has then been obtained by comparing the demodulated bits with the modulated ones.

Fig. 3 shows the results of these simulations for three different $S F_{\text {ref }}$ values as an example. The curves represent the error probability for one selected $S F_{\text {ref }}$ against all the possible $S F_{\text {int }}$. From the figure, it can be easily recognized that for each $S F_{\text {ref }}$ there exists a minimum SIR threshold below which the success probability starts degrading (high BER). Furthermore, the smaller the interfering SF, the higher the SIR threshold required for obtaining an acceptable BER.

Table I summarizes the SIR thresholds leading to a BER of approximately $1 \%$. In the table, we also consider the case when the interfering signal has the same SF of the reference signal. As also documented in the Semtech specifications, LoRa modulations achieve a very high probability of capture effects even with low SIR values (0dB for the different SFs in our simulations, versus $6 \mathrm{~dB}$ specified in [9]). In other words, it is very likely that in case of collisions between two signals modulated with the same SF, the strongest signal can be correctly demodulated. Note that, as the BER curves are very steep, the corresponding Packet Error Rate (PER) thresholds result very similar. 


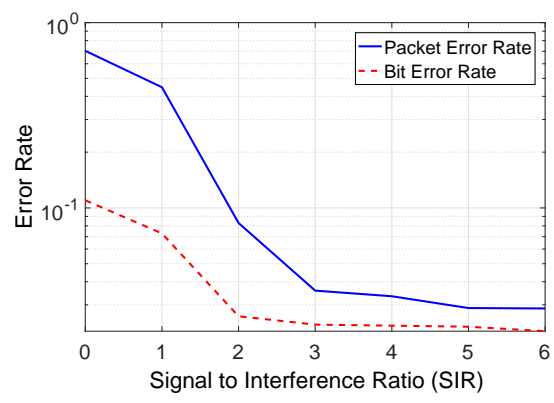

Figure 4. Error rate of the SX1272 transceiver for reference and interferer streams modulated with $S F=7$.

\section{B. USRP experiments}

For validating the thresholds found with the MATLAB simulator, we performed a number of experiments on real LoRa links. To this purpose, we used a Semtech SX1272 transceiver, controlled by an Arduino Yun, for characterizing the behavior of a commercial receiver in presence of collisions. We implemented a LoRa synthesizer able to encode, modulate and generate the I/Q samples of a real LoRa packet, which can be easily transmitted over the air with a USRP B210 board through GNU radio. With this LoRa synthesizer, we generated two traces (one for the interferer and one for the reference LoRa link) for each combination of SFs, composed of a stream of 20 byte-long packets (for the reference SF) and adjusting the payload length of the interfering SF to match the length of the reference signals. The offset of each interfering packet, overlapped in time to the packets of the reference link, has been randomly selected within a window which guarantees that the two packets collide for at least one symbol. We filled the payload of all frames with randomly generated bytes, except for the two bytes that specify the destination address and the payload length. In particular, we assigned the destination address of the SX1272 receiver only to the packets of the reference link. This allows the receiver to discard the interfering packets when they are modulated with the same SF of the reference ones. Finally, we scaled the amplitude of the interfering packet stream to achieve the desired SIR and added it to the reference stream. This correctly models the channel effects when both the reference and interfering transmitters are experiencing a LOS propagation (or NLOS with only one resolvable path), with minimal (or negligible) frequency selective fading 1. For each couple of $S F_{\text {ref }}$ and $S F_{\text {int }}$, the resulting combined stream was transmitted through the USRP towards the SX1272, thus emulating the traffic generated by two different transmitters.

Fig. 4 shows the error rate of the receiver when both the interferer and the reference packets are modulated with $\mathrm{SF}$ equal to 7 . We can observe that, if the power of the reference stream is at least $3 \mathrm{~dB}$ higher than the interferer, the PER is below 2\%. The BER, instead, is very low also when the interferer and the reference packets have equal power. Furthermore, we can observe that the PER cannot be simply obtained as $1-(1-B E R)^{P \cdot 8}$, being $P$ the number of transmitted bytes, because only a sub-set of symbols are

\footnotetext{
${ }^{1}$ In this scenario we verified that, by emulating a multipath channel, selective fading has an impact on the SIR thresholds of about 1 or $2 \mathrm{~dB}$.
}

Table II

SIR THRESHOLDS WITH SX1272 TRANSCEIVER.

\begin{tabular}{|r|r|r|r|r|r|r|}
\hline$S F_{\text {ref }}$ & 7 & 8 & 9 & 10 & 11 & 12 \\
\hline 7 & 1 & -8 & -9 & -9 & -9 & -9 \\
\hline 8 & -11 & 1 & -11 & -12 & -13 & -13 \\
\hline 9 & -15 & -13 & 1 & -13 & -14 & -15 \\
\hline 10 & -19 & -18 & -17 & 1 & -17 & -18 \\
\hline 11 & -22 & -22 & -21 & -20 & 1 & -20 \\
\hline 12 & -25 & -25 & -25 & -24 & -23 & 1 \\
\hline
\end{tabular}

corrupted by the overlapping interfering signal due to the random overlapping of packet transmissions. The results of the experiments are summarized in table III for a subset of reference and interfering SF combinations. The table shows that the SIR thresholds for correct demodulation are similar to the ones obtained in MATLAB simulations and very different (over $10 \mathrm{~dB}$ - an orders of magnitude) lower than the ones in [7], with values as low as $-8 \mathrm{~dB}$. Such power difference between two radio signals can easily appear in common LoRa application scenarios, thus contradicting the common belief that different SFs can be considered as orthogonal in practice.

\section{CONCLUSION}

In this letter we have shown that, because of imperfect orthogonality between different SFs, a LoRa network cell cannot be studied as a simple super-position of independent networks working on independent channels. Indeed, when the power of the interfering signal significantly overcomes the reference signal, the correct demodulation of the reference signal can be prevented. Our experimental results show that on average the co-channel rejection threshold is $16 \mathrm{~dB}$. This power difference can easily appear in near-far conditions, when the interferer is much closer to the LoRa receiver, or when multiple interfering signals are received simultaneously. Implications of imperfect orthogonality and channel captures on network planning are still under investigation. For example, allocating higher SFs, characterized by lower receiver sensitivities, to far users could not necessarily improve their link capacity in case of congested networks. Indeed, higher SFs could be more prone to collisions due to longer transmission times.

\section{REFERENCES}

[1] Worldwide connected devices forecast WWW.statista.com

[2] Semtech. LoRa Modulation Basics. AN1200.22, Revision 2, May 2015.

[3] O. Bernard, A. Seller, N. Sornin, "Low power long range transmitter", European Patent Application EP 2763321 A1 by Semtech Corp., 2014

[4] M. C. Bor, U. Roedig, T. Voigt, and J. M. Alonso, "Do LoRa Low-Power Wide-Area Networks Scale?", In Proc. of MSWiM 2016.

[5] B. Reynders, W. Meert, S. Pollin, "Range and coexistence analysis of long range unlicensed communication", In ICT 2016, Thessaloniki.

[6] B. Reynders, S. Pollin, "Chirp spread spectrum as a modulation technique for long range communication”, In SCVT 2016, Mons, pp. 1-5.

[7] C. Goursaud, J.M. Gorce, "Dedicated networks for IoT: PHY / MAC state of the art and challenges", in EAI endorsed trans. on IoT, 2015.

[8] http://lora.tti.unipa.it

[9] Semtech Corporation, "LoRa SX1272/73 Transceiver Datasheet", 2015.

${ }^{2}$ In table 1 of [7], the lower triangular part follows the law $10 \cdot \log _{10}\left(2^{S F_{\text {ref }}}\right)$, i.e. the SIR thresholds are equal to the spreading gain of a matched filter receiver over an AWGN channel. However, this result is unrealistic, because the receiver does not work by comparing the mean squares of the signals and the interfering signal is not a white process (see for example figure 2). 